\title{
Germanica
}

\section{Le naturisme « fonctionnel » sous Weimar}

Die Neusachliche Instrumentalisierung der Freikörperkultur in der Weimarer Republik

\section{Marc Cluet}

\section{OpenEdition}

Journals

Édition électronique

URL : http://journals.openedition.org/germanica/2398

DOI : 10.4000/germanica.2398

ISSN : 2107-0784

\section{Éditeur}

Université de Lille

\section{Édition imprimée}

Date de publication : 31 décembre 1991

Pagination : 271-290

ISSN : 0984-2632

\section{Référence électronique}

Marc Cluet, «Le naturisme « fonctionnel » sous Weimar », Germanica [En ligne], 9 | 1991, mis en ligne le 17 juillet 2014, consulté le 06 octobre 2020. URL : http://journals.openedition.org/germanica/2398 ;

DOI : https://doi.org/10.4000/germanica.2398

Ce document a été généré automatiquement le 6 octobre 2020.

(c) Tous droits réservés 


\title{
Le naturisme « fonctionnel » sous Weimar
}

\author{
Die Neusachliche Instrumentalisierung der Freikörperkultur in der Weimarer \\ Republik
}

Marc Cluet

1 L'identification idéologique du mouvement, ou plutôt des mouvements naturistes, en Allemagne, pendant l'entre-deux-guerres, est plutôt floue dans les esprits en France. On observe un phénomène de réduction arbitraire, à caractère variable, de la diversité existante. Selon La vie quotidienne sous la République de Weimar (1983), ces «pratiques naturistes » avaient cours dans les " associations de jeunesse de gauche »; par contre, selon l'Histoire de la pudeur de J.-C. Bologne (1986), il s'agissait d'un «nudisme nordique » qui avait « épousé les idées nationalistes et antisémites du prénazisme »․ La thèse, plus ancienne, de M.A. Descamps sur Le nu et le vêtement esquive le problème en diluant toute opinion politique précise dans le mondialisme tendanciel qui serait inhérent au naturisme en tant que tel ${ }^{2}$. Moyennant quoi, il omet de préciser que Richard Ungewitter (1868-1958), fondateur, en 1906, de la première association naturiste au monde, Tefal (Treubund für Aufsteigendes Leben), était un teutomane de la pire espèce, et qu'Adolf Koch (1897-1971), fondateur, en 1924, à Berlin, d'une école de culture physique populaire (et naturiste) était membre du S.P.D. ${ }^{3}$.

2 Les travaux, effectués en Allemagne même, sur l'histoire de "son » naturisme sont plus précis au plan idéologique. Ainsi, Giselher Spitzer distingue dans sa thèse de $1982^{4}$ entre une tendance «völkisch», une tendance "prolétarienne» et une tendance " apolitique », laquelle serait née dès avant 1914, aurait progressé rapidement pendant l'entre-deux-guerres, au détriment des deux autres, pour les absorber finalement après 1945. Ce schéma paraît cependant problématique sur un point (au moins) : le naturisme «apolitique » de l'après-guerre noue-t-il purement et simplement avec le naturisme « apolitique » sous Weimar? n'y aurait-il jamais eu un naturisme spécifique à Weimar, mort avec - ou même avant - la première république allemande, à l'instar de sa « Nouvelle Architecture »? En effet, si l'apolitisme signifie généralement la mise entre parenthèses de la politique, au nom du pluralisme démocratique, sous Weimar il a pu 
signifier aussi la négation active (et illusoire) de la politique, au nom de l'idéologie de la " désidéologisation », la fameuse "Nouvelle Objectivité ». C'est ce naturisme perdu que nous nous proposons de rechercher, en nous gardant, par avance, de vouloir prétendre que tous les naturistes modérés de Weimar - voire même simplement une majorité d'entre eux - y aient été ralliés. En effet, le refus des extrêmes ne saurait impliquer en soi la « désidéologisation » active, surtout chez les naturistes, dont le mouvement puise, à ses origines, dans une mystique «néo-romantique» de la nature et, plus particulièrement, du soleil. D'un autre côté, tout naturiste est, par définition, enclin à l'« Objectivité » sur un point précis : il est persuadé qu'une "valeur» au moins mérite d'être "liquidée», la pudeur, qui, du fait du champ sémantique élargi de l'allemand «Scham», touche potentiellement à la morale (cf. Nietzsche).

3 Dans ces conditions, il conviendra d'opérer, dans notre quête du naturisme «fonctionnel ", à l'aide du concept de perméabilité, plus ou moins grande, du naturisme de l'entre-deux-guerres aux théories de la « Nouvelle Objectivité ». Cette perméabilité sera maximale chez les Kulturphilosophen ralliés, à la fois, à la " démocratie athlétique " et au naturisme, tels le romancier et essayiste Frank Thieß ou le psychologue du travail et des sports Fritz Giese ${ }^{6}$. Au contraire, il s'agira d'une (quasi-)imperméabilité aux théories de la «Nouvelle Objectivité chez les théoriciens du naturisme «völkisch» ou "prolétarien", qui, à ce titre, sont exclus de notre champ d'investigation. Celui-ci recouvrira un nombre limité d'auteurs importants ou d'équipes rédactionnelles de revues importantes. On retiendra :

4 1) Adolf Koch pionnier du naturisme populaire, déjà évoqué, et ses collaborateurs, notamment son «bras droit», le médecin Hans Graaz, qui s'exprimaient principalement dans la revue officielle de la Kochschule, Körperbildung /Nacktkultur. Même si Koch a pu prétendre, à ses débuts, que l'un de ses buts était de renforcer la combativité du prolétariat en vue de la lutte des classes, le ferment réformiste que recelait son programme - l'idée d'une simple remise en forme des plus défavorisés (en dehors de toute considération tactique) - a vite pris le dessus. Finalement, l'anticapitalisme s'est limité ici à la dénonciation des fabricants de spiritueux («Alkoholkapital») ${ }^{7}$. Très significativement, de l'aveu même de Koch, sa démarche était jugée "bourgeoise» par une «jeunesse dressée à réciter des slogans de parti politique $»^{8}$, et, par ailleurs, il s'est vu accuser par un Kulturkritiker fascisant de prôner une "éthique utilitariste" et une "ligne de vie [Zuchtleben] eudémoniste ", très américaine d'inspiration'.

2) Hans Surén (1885-1972), ancien directeur de l'École Supérieure d'Éducation Physique de l'Armée (jusqu'en 1924), propagandiste infatigable du naturisme sous toutes ses formes, solitaire ou en groupe, unisexe ou mixte, oisif ou sportif, mais avec une certaine prédilection pour la gymnastique nue. Son «travail» fut salué avec emphase par Thieß et Giese : «sinnvolle und präzise Körperkultur», «echte Sportkultur» ${ }^{10}$. - Bien qu'il se soit bruyamment rallié au nazisme, avec honneurs à la clef dès 1932, il n'y a guère d'éléments annonçant cette "conversion" dans ses écrits de la période weimarienne. G. Spitzer, qui les a passés au crible pour y déceler d'éventuels éléments «völkisch», signale surtout l'anti-intellectualisme, et arrive à la conclusion qu'« en aucun cas, il ne saurait être considéré comme fortement engagé à droite $»^{11}$. Personnellement, je décèlerais même chez lui, à cette époque, des éléments presque incompatibles avec le nazisme, tels son injonction à se libérer de la discipline sportive 
(Drill) pour "vivre sa personnalité» («sich selbst erleben»), ou encore son espoir de voir les vols transatlantiques contribuer à « la compréhension mutuelle des peuples $»^{12}$.

3) L'équipe rédactionnelle de Licht-Land, organe officiel d'une fédération naturiste apolitique, au sens du pluralisme démocratique, Liga für freie Lebensgestaltung, qui, par le truchement d'une théoricienne naturiste hors de pair, issue du Wandervogel, Therese Mülhause-Vogeler (1893-1984), adopta des positions nettement antinazies, et proweimariennes, au début des années trente ${ }^{13}$. «Ligue» et revue établirent d'ailleurs des contacts amicaux avec des naturistes français du Midi ${ }^{14}$.

7 4) Les équipes rédactionnelles des revues Figaro et Sorna, qui alliaient naturisme et érotisme. Ces revues, qui n'avaient pas de véritables organisations derrière elles, cultivaient l'ambiguïté à des fins commerciales. Il parait cependant probable qu'elles touchaient non seulement un public d'amateurs "avertis", mais aussi de naturistes " avancés d'esprit » et réfractaires à la vie de groupe. En effet, l'érotisme n'avait pas besoin de la caution naturiste pour s'afficher sous Weimar. Les articles, de tenue inégale, témoignent d'un souci constant d'incarner le progrès en matière de mœurs et de mode. La formule journalistique - étrange aujourd'hui - de ces revues pouvait répondre aux désirs d'un public naturiste marginal, que Licht-Land définit, un jour, de la sorte - par souci de se démarquer: «sentationslüsterne AuchNacktkultur»" ${ }^{15}$. Compte tenu de ses liens potentiels avec la "Nouvelle Objectivité", en tant que produit commercial ${ }^{16}$, il fallait l'inclure ici.

8 Nos auteurs et rédacteurs pronaturistes sont unanimes à penser que l'exposition du corps au vent, au soleil et à l'eau, voire simplement à l'atmosphère ambiante d'un gymnase $^{17}$, constitue un facteur de santé si puissant qu'il serait dommage d'y soustraire la moindre parcelle de peau ${ }^{18}$. Les arguments d'origine "néo-romantique» des premiers théoriciens naturistes, qui escomptaient un regain de vitalité par infusion de "forces naturelles", cèdent généralement le pas à diverses théories de l'hygiène prophylactique. Chez Mülhause-Vogeler, on perçoit certes encore quelques relants de philosophie de la nature stoïcienne, quand elle estime, par exemple, que l'exposition du corps aux éléments "soutient le travail [Arbeit] de ses forces curatives " ${ }^{19}$; mais rien de tel chez un $\mathrm{D}^{\mathrm{r}}$ Graaz. La peau est un "organe important ", car elle sert à éliminer les déchets du métabolisme et les toxines; des "découvertes très récentes " tendraient même à lui assigner le rôle primordial d'« organe de contrôle et de régulation » du système endocrinien. Seule son exposition totale, en particulier à la stimulation lumineuse (Lichtreiz), lui permettrait de fonctionner «à plein rendement » («zur vollen Leistungsfähigkeit gebracht») ${ }^{20}$, et d'entraîner ainsi, du fait de son rôle régulateur, l'organisme tout entier sur la voie de la meilleure santé.

9 Or, selon l'obsession de l'économique, propre à l'époque, et à l'idéologie de la « Nouvelle Objectivité», la santé, c'est aussi de l'argent. Graaz précise que les hommes, ainsi «rendus efficaces [leistungsfähig] (...) ne compromettent pas leur situation économique par la maladie $»^{21}$. Bien évidemment, par là même, ils épargnent non seulement leur budget personnel, mais - «l'économie globale» (Gesamtwirtschaft). Tous nos auteurs et rédacteurs, excepté peut-être Mülhause-Vogeler, tendent à souligner cet avantage du naturisme. Dans un article de la revue Sorna sur La culture physique [nue] et ses implications économiques, un certain Rudolf Teßmann donne à penser à ses lecteurs que «les adeptes de la culture physique [nue] ne représentent pas une charge pour les caisses d'assurance-maladie ", dans la mesure où ils ne solliciteraient guère leurs prestations. Cette contribution indirecte serait d'autant plus précieuse que 
les caisses souffriraient de déficits chroniques, en dépit des cotisations élevées qu'elles perçoivent ${ }^{22}$. Surén, pour sa part, se plaît à rêver du "bénéfice inouï » qu'apporterait à un peuple, du fait de ses vertus prophylactiques, le naturisme généralisé - que ce peuple s'adonne à l'industrie et au commerce ou... à la guerre («Kriegshandwerk») ${ }^{23}$.

Cependant, d'après le $\mathrm{D}^{\mathrm{r}}$ Graaz, le bénéfice visible de la prophylaxie naturiste représenterait tout juste "la moitié » du bénéfice réel. En effet, il croyait avoir découvert la cause de l'asthénie fonctionnelle dont souffrent beaucoup de citadins : la " maladie la plus courante », aux symptômes multiples, dont la baisse de la capacité de travail, serait tout simplement due à la mauvaise irrigation sanguine des tissus cutanés ("l'anémie périphérique »). Frictions et surtout naturisme seraient donc des remèdes tout indiqués contre ce mal que les caisses d'assurance-maladie seraient obligées de nier, faute de moyens financiers, en limitant arbitrairement les congés pour fatigués ${ }^{24}$. Les naturistes, eux, ont du ressort. Surén et Mülhause-Vogeler en sont également convaincus, en dehors de toute explication scientifique, puisque tous deux affirment que le naturisme arme l'individu contre les soucis quotidiens et les revers de l'existence, qui accablent, voire paralysent les dépressifs ${ }^{25}$.

11 Toutefois, les bienfaits sanitaires et économiques du naturisme ne se limitent pas là : tous nos auteurs et rédacteurs conviennent qu'il est un auxiliaire puissant à la régénération de la force de travail. Cette vertu régénératrice vaudrait plus particulièrement pour le travail à la chaîne ou en atelier vétusté, sale et sombre, mais concernerait aussi, selon un article de la revue Licht-Land, "la vendeuse débitant sa litanie au client derrière un comptoir infesté de microbes » et "l'employé plongé six jours sur sept dans ses livres de comptes bourrés de chiffres $»^{26}$. À tous ceux-là, le weekend naturiste procurerait une force de travail sans cesse renouvelée. Au printemps 1929, la revue Figaro publiait un montage de deux photos, sans doute encore plus éloquent à cet égard que les développements habituels sur l'élimination des déchets physiologiques (Schlacken) dans le naturisme oisif et « l'affranchissement du rythme de mouvement mécanique [imposé par les machines]», dans la gymnastique nue ${ }^{27}$. La première photo présente une jeune ouvrière derrière un engin énorme (une rotative ?), la seconde, un couple nu en promenade au bord d'un ruisseau - le tout assorti de cette légende : « La nature et le naturel aident à surmonter les effets nocifs du travail ${ }^{28}$.

Ainsi adapté au mieux aux conditions de production modernes, le naturiste aurait tout pour réussir au plan professionnel. Très significativement, au début de la crise économique en Allemagne, alors que le chômage commençait à exacerber la concurrence entre salariés, la "Ligue » faisait sa propre publicité sur le thème du naturisme, facteur de réussite :

Höchste Leistungsfähigkeit ist die Losung, wenn man es im Leben zu etwas bringen will. Höchste Leistungsfähigkeit aber kann nur sein im gesunden Körper, dem seine Erholungszeit auch wirklich als Erholung bewilligt wird. (...) Willst du nicht ein solcher Mensch sein? Ja? Dann komm herein in die Reihen der Freikörperkultur! $(. . .)^{29}$.

Cependant, l'essentiel de la propagande naturiste visant plutôt à se faire accepter qu'à recruter de nouveaux adeptes, l'argumentation économique autour de la productivité des naturistes se situe généralement au niveau de la société tout entière. Ainsi, selon la formule de Teßmann, ils constituent «un plus » («ein Plusfaktor») pour l'économie ${ }^{30}$. $\mathrm{Au}$ dire de Graaz, le naturisme rendrait même davantage de services à ce titre que le sport, qui pourtant était systématiquement vanté, et exploité, sous Weimar, pour ses vertus régénératrices ${ }^{31}$. En effet, à la différence du sport, qui, d'après Graaz, ne 
concernerait a priori que ceux qui ont déjà par eux-mêmes le plus de ressources physiques («die Leistungsfähigeren»), le naturisme, en particulier dans ses variantes oisive et "gymnique douce ", rendrait de semblables services de régénération auprès de tous ceux qui, sans cela, vivraient « au jour le jour, de façon naïve et primitive »32.

Est-ce à dire que le naturisme, tel que le conçoit Graaz, doive rester enfermé dans les limites biologiques d'individus qui ne sont pas les plus valides? - Nullement! D'une part, ces limites, on l'a vu, sont repoussées, dans une certaine mesure, par la pratique même du naturisme. D'autre part, Graaz, à l'instar de tous ces collègues théoriciens, est convaincu que le naturisme œuvre dans le sens de l'eugénisme, qu'ils appellent de leurs vœux. La nudité partagée amènerait les individus à reporter leur souci de l'apparence extérieure («Gefühl für Exterikultur») sur leur condition physique réelle, et, par là même, à accorder de l'importance, dans le choix de leur partenaire, à des critères biologiques $^{33}$. Plus concrètement, un Surén voit dans le naturisme une véritable école pratique du jugement anthropologique: les femmes et les filles $\mathrm{y}$ apprendraient à accorder leur préférence aux "jeunes gens halés à la poitrine large et à l'œil vif ", plutôt qu'aux dandys malsains; et les hommes, quant à eux, à faire abstraction des vêtements à la dernière mode, chez les femmes, au profit de leur condition physique ${ }^{34}$. Ainsi, de façon générale, les naturistes tendraient à " adopter une hygiène consciente de la procréation » du plus grand intérêt économique à long terme. En effet, à mesure que disparaîtrait la part de «matériau humain délabré » («anbrüchiges Menschenmaterial») ${ }^{35}$ dans la société, «non seulement l'économie publique, mais bien plus encore la rentabilité [Wirtschaftlichkeit] de l'individu » seraient dynamisées ${ }^{36}$.

Si l'optimisation génétique des couples naturistes est du plus haut intérêt pour la société, en revanche, leur optimisation au plan relationnel, qui serait aussi du ressort du naturisme, semble devoir profiter essentiellement aux intéressés eux-mêmes. En effet, chez les naturistes, on ne rencontrerait pas de "nœuds de vipères " affectifs, dans la mesure où des partenaires, qui se sont fréquentés en l'état de nudité, avant de se lier, sauraient éviter les méprises dues au désir d'ascension sociale ou à l'attirance strictement sexuelle. On conçoit que le facteur social soit (prétendument) neutralisé, dès lors que les vêtements sont tombés. Nous aurons l'occasion d'y revenir. - Mais pourquoi le sexe? Tous nos auteurs et rédacteurs en conviennent pourtant sans exception : la nudité offerte en permanence éliminerait les fantasmes érotiques autour de la «terra incognita» de l'autre sexe, et, de ce fait, la formation de couples sur cette base incertaine. On ne se marierait plus pour savoir enfin. Avant formation d'un couple, au stade de la découverte mutuelle, la relation à l'autre, en tant qu'être sexué, serait paradoxalement asexuée, " objective » («natürlich und sachlich», «herbkühl») 37, si bien que cette découverte mutuelle pourrait se concentrer sur la personnalité de l'autre. De tels couples, qui, outre les critères eugéniques, se formeraient en fonction d'affinités réelles, que Koch prétendait d'ailleurs aider à découvrir par l'enseignement de notions de caractérologie $e^{38}$, non seulement «fonctionneraient bien» («reibungslos») $)^{39}$, mais contribueraient aussi, comme l'observe Mülhause-Vogeler, au bon fonctionnement de l'économie. En effet, les enfants issus de ces couples soudés par la psychologie tendraient à constituer une "force de travail efficace» («leistungsfähige Kräfte»), plutôt qu'à devenir des «inadaptés (...) à la charge des organismes de l'assistance publique $»^{40}$.

16 La modification des rapports de couple, induite par le naturisme, devait s'accompagner, du vœu de nos auteurs et rédacteurs, de la recherche de formules juridiques nouvelles 
allant dans le sens de Vopen marriage, proposé alors par l'Américain Ben B. Lindsey, juge pour mineurs à Denver. Sa proposition d'officialiser l'union libre, abondamment discutée en Allemagne sous l'appellation séduisante de «Kameradschaftsehe»", semblait bien accordée, non seulement au "partenariat " naturiste, que MühlhauseVogeler qualifiait de «kameradschaftliches Gleichstreben»" mais encore à la camaraderie généralise que le naturisme était censé instaurer entre hommes et femmes à l'échelon de l'organisation naturiste, voire de la société tout entière. Ce thème est surtout développé par Frank Thieß, dans le prolongement des théories de MülhauseVogeler (non citée), ainsi que d'une autre ancienne du Wandervogel, Elisabeth Busse Wilson (expressément citée) ${ }^{43}$ : à terme, la " désérotisation » opérée par le naturisme aboutirait, avec le renfort non négligeable de l'éducation mixte et de l'émancipation féminine, à arracher la femme à son rôle de proie (ou de piège), et l'homme, à son rôle de chasseur (ou de "pigeon »), au profit de relations normalisées dans un « esprit de camaraderie entre égaux » («Geist gleichgeordneter Kameradschaftlichkeit» ${ }^{44}$ ).

Cependant, par delà la disparition de la polarité traditionnelle des sexes masculin et féminin, tous les rapports sociaux seraient aplanis par le naturisme, au moins le temps du week-end, voire durablement, selon les plus hardis parmi nos auteurs et rédacteurs. Même Surén, ancien militaire, et officier supérieur, s'abandonne volontiers à ce miracle de la nudité partagée : « Hier - non, ce matin même - on était encore le chef d'un grand nombre, et voici qu'on est un camarade parmi d'autres [gleichgestellt] $»^{45}$. Le naturisme brasserait les couches sociales, "de l'aristocratie au prolétariat », et, selon un rédacteur de Licht-Land, se référant probablement à la "Ligue», on y verrait «le directeur de banque, le magistrat, le médecin jouer au ballon, en toute innocence [harmlos], avec le manœuvre ou l'ouvrier $»^{47}$. Seuls Koch et ses collaborateurs évitent ce type de discours, sans doute parce que la Kochschule recrutait pour l'essentiel dans les milieux populaires, et qu'aucun brassage social n'y avait donc lieu. Cependant, les attentes de Koch quant à une éventuelle homogénéisation de la société tout entière, à travers le naturisme, sont certainement aussi fortes que chez ses collègues théoriciens - à l'exception, cette fois, de Surén, qui postule la prééminence sociale des gymnastes naturistes, véritable «aristocratie du biceps $»^{48}$. Koch et les autres prétendent que les naturistes, tout en affirmant chacun leur personnalité propre, réalisent nonobstant l'homme générique, réputé bon et sociable. «Mensch überhaupt», "reinmenschlicher Mensch», «Bruder Mensch», «Gemeinschaftsmensch» sont les expressions qui reviennent sous leur plume ${ }^{49}$.

Cette mutation fondamentale entraînerait, tout d'abord, une sociabilité informelle d'un type nouveau - entre le chauve et le chevelu, la garçonne et le bambin ${ }^{50}$ - dont un rédacteur de Licht-Land donne la formule magique, vaguement goethéenne : «ein Reich des Ausgleichs aller Gegensätze (...), wo man Mensch, wirklich Mensch sein darf» / « un royaume où tous les antagonismes sont abolis (...), où on peut être homme, vraiment homme»" ${ }^{51}$. Quand Kracauer décrit «le public homogène de la métropole qui [serait] à l'unisson, du directeur de banque au commis de bureau, de la star à la dactylo»" ${ }^{52}$, les formulations sont assurément plus brillantes; pourtant, c'est la même illusion, caractéristique de l'époque, et de l'idéologie de la "Nouvelle Objectivité ", qui est à l'œuvre: le rythme de l'homogénéisation sociale à partir de loisirs "tout public ", cinéma ou « libre culture ».

Les implications socio-politiques, plus lointaines, de la "Nouvelle Homogénéité ", induite par le naturisme, ne sont, elles, que rarement abordées, et ce, uniquement dans 
les écrits de la Kochschule et dans la revue Sorna, Un article de cette dernière, au titre éloquent, Die Gemeinschaft, d'un certain Rudolf Karl, annonce que la société, remodelée par le naturisme, ne connaîtrait plus d'institutions marquées du sceau d'une classe ou d'une élite spécifiques («mit Klassenoder Standescharakter»), mais seulement des institutions "à caractère communautaire $»^{53}$. Koch, pour sa part, imagine que tous les mondes parallèles («Unzahl von Welten»), où nous vivons présentement dans la séparation, pourraient et devraient s'évanouir, au profit des seules structures culturelles et économiques («Gliederungen nach Volksund Wirtschaftskörpern») ${ }^{54}$. Dans le même ordre d'idées, Graaz appelle de ses vœux «l'organisation fonctionnelle [zweckmäßig] des pouvoirs politiques et économiques $»^{55}$. Chez Karl, c'est l'autorité même de l'État qui est remise en question: "Les hommes socialisés [Gemeinschaftsmenschen] ne se laissent rien dicter d'en-haut», si bien que "le principe de l'autorité fait place à la gestion [Verwaltung] $»^{56}$. Dans ces conditions, la politique, au sens traditionnel du terme, n'a évidemment plus lieu d'être. Koch condamne les partis en tant que "mondes " séparés; Graaz, dans le cadre d'une critique globale, et "objectiviste», de la mentalité magique («animistisches Weltbild» $)^{57}$, qui mérite qu'on s'y attarde.

Au nom de la science exacte («wissenschaftliche Weltbetrachtung», «wissenschaftliches Weltbild»), Graaz s'en prend à la fois à la politique et... au vêtement. Les luttes politiques contemporaines en Allemagne s'articuleraient essentiellement autour d' emblèmes, investis positivement par leurs protagonistes respectifs, et négativement par leurs antagonistes respectifs. Pareil «fétichisme»- lourd de conséquences, puisque, par sa faute, « nous continuons à nous défoncer mutuellement le crâne »- remonterait, en fait, à l'époque barbare où les guerriers portaient, à l'avant de leurs troupes, une perche garnie d'une tête d'animal, dont ils croyaient tirer de la force. Or, il y aurait là un parallélisme avec l'institution - qui perdure hélas! - du vêtement. D'après des observations ethnologiques, celui-ci aurait été inventé pour se protéger, non du climat, mais... de l'agression de mauvais esprits. Moyennant quoi, "désidéologisation » et naturisme sont indissociablement liés dans l'esprit de Graaz, puisque c'est la même rationalité, triomphant du « fétichisme » politique et vestimentaire, qui permettra leur réalisation simultanée. - Chez Karl, cette même concomitance se passe de démonstration laborieuse, dans la mesure où "nu » est, pour ainsi dire, synonyme, chez lui, d'« objectif » (au sens de la « désidéologisation ») - quand il écrit par exemple : « Le mouvement naturiste, qui observe et juge toute la vie sociale à nu [nackt], a pour fin dernière de réaliser la communauté ${ }^{58}$.

21 Il y a tout lieu de croire que cette communauté décloisonnée et rationalisée, dont rêve une minorité hardie d'auteurs et de rédacteurs pronaturistes, serait très largement pacifiée, exempte de conflits. En effet, d'une part, on vient de le voir, la " politique » se limiterait désormais à la "gestion », aux mesures d'ordre technico-organisationnel, et, par ailleurs, surtout, le naturisme «institutionnel» ne manquerait pas d'absorber les forces «chaotiques» de l'homme. C'est Koch, le théoricien de sensibilité socialdémocrate, qui paradoxalement insiste le plus sur la fonction de défoulement du naturisme. "Nous faisons beaucoup les fous», dit-il, «nous aimons faire les fous ${ }^{59}$. Selon un de ses collaborateurs, ce serait le juste «droit » des ouvriers, à qui on a volé leur jeunesse, de se livrer aux jeux les plus animés et, selon le maître lui-même, ce serait là une manière spécifique, pour les ouvriers, de compenser le travail sur machine ${ }^{60}$. Ils disposeraient ainsi d'un moyen, sûr et paisible, de se soustraire à « un monde "qui, trop souvent, n'offre d'autre issue que de crier son émotion chaotique" » 
(«eine Welt, "deren einzige Steigerung oft genug nur ein leidenschaftliches chaotisches Schreien ist"». Cette formule, reprise d'un autre (non cité), traduit bien, à travers ses accents anti-expressionnistes et anti-révolutionnaires, l'effet de stabilisation induit par le naturisme « fonctionnel ».

Cependant, le défoulement n'en est sans doute pas la seule composante stabilisatrice. Si on prend à la lettre la théorie de Koch, selon laquelle il y aurait continuité entre l'instinct ludique (Spieltrieb) et l'instinct sexuel ${ }^{61}$, on conçoit aisément que l'épanouissement, promis aux naturistes, auprès de partenaires sur mesure - ou trouvé, à défaut, dans l'auto-érotisme, que ces auteurs et rédacteurs déculpabilisent à peu près totalement ${ }^{62}$ - constitue aussi un puissant facteur de stabilisation. Par le biais de "l'hygiène ", dont la morale devrait reconnaître les " exigences " ${ }^{63}$, l'économie libidinale est mise au service de l'économie tout court. Assez curieusement, Surén se retrouve ici aux côtés des plus hardis, voire à leur tête : «Reinigender Ausgleich der Geschlechter», «(Selbst-)Auslösung», «(Selbst-)Entspannung», «Befreiung und Kräftigung» sont ses termes éloquents - qui correspondent à autant d'étapes d'un processus particulier d'optimisation de la force de travail. Un certain Hans H. Reinsch de la revue Figaro en livre la formule explicite : « La force de travail de l'individu doit être sans cesse ravivée par l'activité sexuelle, sinon elle est engloutie dans une morne routine de bête de somme asexuée ${ }^{64}$.

On ne discerne vraiment aucune source de frictions dans cette société bien huilée. Reste évidemment l'antagonisme fondamental homme/machine, que le naturisme «fonctionnel » ne prétend pas résorber, mais seulement aménager le mieux possible. Aucun de nos auteurs et rédacteurs, du fait probablement des origines "néoromantiques» du mouvement naturiste, ne parvient à aimer sans réserve l'univers des machines. D'un autre côté, il ne saurait être question de les briser. Teßmann estime que ce serait une " grosse erreur de vouloir éliminer au $\mathrm{XX}^{\mathrm{e}}$ siècle la production industrielle et mécanisée», quel que soit son coût humain, qu'il faudrait cependant - et c'est là le sens de son propos - réduire au maximum par la culture physique (nue) ${ }^{65}$. MülhauseVogeler, qui est encore plus déchirée à cet égard, du fait de ses liens avec le Wandervogel, rejoint Teßmann dans le «réalisme ${ }^{66}$. La plaie ouverte par le machinisme, à défaut d'être guérie, peut être aseptisée et pansée - à moins... qu'on ne l'occulte sur le mode cosmétique ! Cette « solution » est si caractéristique de l'esprit de la «Nouvelle Objectivité » que nous ne voudrions pas omettre de la signaler, bien qu'elle soit tout à fait isolée dans notre corpus, et donc assurément marginale. C'est paradoxalement encore dans l'entourage de Koch que cette proposition est avancée : par Wilm Burghardt qui, paradoxe supplémentaire, allait assurer la continuité du naturisme sous le III ${ }^{\mathrm{e}}$ Reich. Dans un article de la revue Körperbildung / Nacktkultur, intitulé Form, Stil, Kunst - und Kultur ${ }^{67}$, et rédigé dans un style lapidaire, qui n'est pas sans rappeler certaines productions "poétiques» antilyriques de la période weimarienne ${ }^{68}$, Burghardt commence par constater qu'il existe une beauté, froide et inhumaine, de la machine, voire de la production elle-même, dès lors qu'elle est taylorisée, relevant de ce qu'il appelle le «style ». Puis, il lui oppose une autre beauté, la beauté corporelle, sous le vocable de « forme ». À première vue, les deux esthétiques, machiniste et "organique", paraissent antinomiques. Toutefois, et c'est là la réconciliation annoncée, une gymnastique de groupe ou une chorégraphie nue serait capable d'opérer la synthèse, qualifiée d'« objective» (« sachlich ») (!), non seulement le temps de son exécution, mais durablement, et à l'échelle de la société tout entière, à travers un processus d'esthétisation globale, dont on discerne mal les modalités, mais 
qui, selon Burghardt, aurait pour effet (accessoire ? !) d'impliquer la «mort de l'art». Cette utopie, qui, abstraction faite du medium artistique utilisé pour l'enclencher, rappelle l'« État esthétique » de Schiller, et anticipe sur la société « libérée » de Herbert Marcuse $^{69}$, prendrait-elle la forme de la pantomine généralisée ? en l'état de nudité ? Burghardt n'en précise rien. - Laissons donc rêver le lecteur à partir de ses termes mêmes :

Der Körper steht im Raum.

Der Körper bewegt sich. Er geht. Zuerst wie auf der Straße. Bald lernt er die Hacken heben, lernt federn, schwingen, lernt hocken, lernt den Rumpf beherrschen (...).

Der Körper sieht die anderen Körper.

So bilden sich Körper. Augen lernen sehen, Körper fühlen.

Körper bilden den Raum - nicht mehr der Raum den Körper. Es wird ein sachlicher

Stil, ohne gemachten Stil. Form und Stil sind aus einer Einheit.

Körper schaffen Werte: im Alltag, im Spiel, im Sprechen, im Singen, in der Bewegung, im Zeichnen, im Werk, im Handeln. - Theater ist uns überflüssig geworden (...).

Wie kennen deshalb keinen Begriff für Kunst. d'abord, le fait même qu'elle a été conçue au sein de la Kochschule, et publiée dans son organe officiel, confirme l'impression - qui s'est probablement déjà imposée au lecteur - comme quoi les théories de la «Nouvelle Objectivité » exercent une fascination particulière sur les esprits formés à la social-démocratie, Koch et ses collaborateurs. Fascination sensiblement plus forte, de toute évidence, que chez un esprit «remué » par le Wandervogel, Mülhause-Vogeler, ou façonné par la Reichswehr, Surén. Ces derniers semblent avoir sélectionné, chacun, surtout un thème de prédilection, dans la palette idéologique contemporaine, en fonction de préoccupations personnelles: elle, l'optimisation des relations de couple; lui, l'équilibre psycho-sexuel de l'individu. En revanche, le naturisme de la Kochschule s'ouvre largement aux conceptions sociopolitiques de la " Nouvelle Objectivité »: « désidéologisation », décloisonnement social, eugénisme à finalité productiviste, sublimation des pulsions « chaotiques » sont inscrits au programme de cette "école ». Nous avons parlé de "paradoxe ", à ce sujet, compte tenu du fait qu'ici, non seulement la révolution cède le pas au réformisme, mais, dans une large mesure, le réformisme lui-même, à une "anthropotechnie » de tendance réactionnaire. Toutefois, pour peu qu'on replace cette dérive droitière dans le contexte global de la social-démocratie allemande de l'entre-deux-guerres, le paradoxe s'évanouit. En effet, comme le montre Helmut Lethen, dans son livre sur la « littérature du socialisme blanc", le S.P.D. lui-même était alors perméable aux théories de la « Nouvelle Objectivité ${ }^{70}$. Ainsi, au congrès du parti de 1927, Rudolf Hilferding brossa un tableau séduisant de la convergence souhaitable, sous les auspices d'un État «neutre» et strictement gestionnaire, entre l'économie planifiée et un capitalisme parvenu à maturité. Le parti essuya, d'ailleurs, à ce titre, semblables reproches, sur sa gauche, que Koch lui-même ${ }^{71}$.

En comparaison de la Kochschule, les revues naturistes "à sensation ", Figaro et Sorna, paraissent certes portées sur les théories à la mode, mais sans jamais atteindre le même degré de "sensationnel », involontaire, qu'un Graaz - quand, par exemple, le plus sérieusement du monde, il voit les partis politiques tomber avec... les maillots de bain ! 
ou assimile implicitement, de par sa terminologie, le simple port de vêtements à... une manie « fétichiste »! La grande différence réside dans le fait que le «modernisme » de ces revues n'est jamais "spéculatif ", mais tend à se limiter aux derniers "progrès " observables. Ainsi, par exemple, l'appel de Teßmann à voir réaliser des institutions sans caractère de classe ni de caste correspond exactement à la réalisation, à l'époque, d'un grand nombre d'équipements collectifs («Gemeinschaftsbauten») : hôpitaux, centres de convalescence, gymnases, piscines, maisons communes, salles de congrès, etc..$^{72}$ Dans le même ordre d'idées, on peut constater que Figaro prit la défense de la jupe courte et des bas couleurs chair en 1929, précisément à l'apogée de cette mode ${ }^{73}$ - le renversement de tendance intervenant à partir de l'été 1930. On a donc l'impression que ces revues «collent» simplement à l'époque, et on en devine la raison: la santé, l'efficacité, l'économie et les utopies correspondantes peuvent certes cautionner le naturisme luimême, mais guère son alliance avec l'érotisme. Pour ce, il faut une légitimation plus large, tel "l'air du temps». D'ailleurs, significativement, on rencontre ce type de "globalisation» dans Figaro: la "redécouverte du corps nu » - on notera l'ambiguïté de l'expression - y est rapportée à « l'époque présente, époque agitée entre toutes " («die heutige Zeit, die Zeit zwischen den Revolutionen $»^{74}$. Pareille stratégie n'exclut cependant pas l'existence réelle d'une minorité naturiste, individualiste et frivole, qui pouvait éventuellement se reconnaitre dans ces publications ${ }^{75}$.

Toutefois, selon toute vraisemblance, il y a au moins un thème, apparaissant dans ce genre de revues, qui devrait échapper au soupçon de n'avoir été que tactique, à savoir la valeur économique du naturisme. En effet, abstraction faite du détail des différentes argumentations, c'est là un thème constant dans l'ensemble de notre corpus, au point d'en constituer, outre la modération politique, une véritable caractéristique. Rien d'étonnant à cela, puisque l'équivalence santé / économie avait très largement cours, à l'époque, dans les milieux « libéraux ». Jusqu'au bourgmestre de Cologne, Adenauer, qui déclarait que les exercices physiques étaient « un médecin au chevet de l'Allemagne »! jusqu'au bourgmestre de Berlin, Böß, qui claironnait : « Ne bâtissez pas d'hôpitaux, ni de sanatoriums, mais des équipements propres à donner de la vigueur et de la santé à notre peuple! $»^{76}$. Il suffit d'ajouter l'épithète «nu» et de broder autour de ces maximes, pour voir se reconstituer, sous nos yeux, des pans entiers de l'édifice théorique de nos auteurs et rédacteurs pronaturistes.

La fragilité de ces théories ressort du fait qu'en 1931 Hjalmar Schacht put retourner contre la "démocratie athlétique " l'argumentation économique dont elle était coutumière; or, on aurait pu procéder à l'identique contre le projet - à vrai dire, globalement marginal - d'une "démocratie naturiste ». D'après lui, l'Allemagne se serait ruinée par les « dépenses somptuaires » de ses communes sur des «équipements de luxe », tels des stades, des piscines, des espaces verts, etc., qui « n'ont dégagé aucun profit économique » [wirtschaftliche Rente], et n'en dégageront jamais $»^{77}$. Cependant, la principale menace qui pesait alors sur la "démocratie athlétique ", et naturiste, résidait, sans doute, dans sa propre réceptivité aux théories nazies. La carrière de Burghardt le prouve peut-être encore davantage que celle de Surén, qui n'était pas simplement prédisposé par son naturisme "fonctionnel ", mais aussi par sa condition d'ancien officier de la Reichswehr. Toutes proportions gardées, l'ambition de Burghardt d'opérer une synthèse sociale à partir de la chorégraphie annonce la " régie de masses " du III ${ }^{e}$ Reich et, en particulier, le cérémonial de Nuremberg, qui avaient pour enjeu symbolique de visualiser la transformation totalitaire de la sociétée ${ }^{78}$. De façon plus générale, l'instrumentalisation de l'homme au service de l'économie préfigure son 
instrumentalisation à des fins impérialistes, ou "biopolitiques » - ces dernières étant même déjà servies de facto par l'eugénisme à finalité productiviste d'un Graaz.

\section{NOTES}

1. - Cf. Lionel Richard, La vie quotidienne sous la République de Weimar, Paris, 1983, p. 231, et J.C. Bologne, dito, 1986, p. 329 et p. 341.

2. - Cf. Marc-Alain Descamps, Le nu et le vêtement, Paris, 1972, passim. Egalement, résumé de la thèse, in : Idem, L'invention du corps, Paris, 1986, p. 90.

3. - Cf. Idem, Le nu..., p. 128 sq., et, pour l'identification politique d'Ungewitter et de Koch, Giselher Spitzer, Der deutsche Naturismus, Ahrensburg, 1983, p. 81 sqq. et p. 141 sqq.

4. - Cf. n. 3.

5. - Nous proposons ce terme par référence à la gymnastique "fonctionnelle ", développée à l'époque. De par sa finalité strictement pratique et «civile», elle s'opposait à la fois à la gymnastique acrobatique et à 1 '« entraînement " militaire (Jahn et ses continuateurs de l'ère wilhelminienne). Ses applications vont de la gestuelle quotidienne (se baisser correctement) à l'ergonomie, en passant par l'accouchement sans douleur. - On notera que la plupart de ses protagonistes souhaitaient que les exercices fussent exécutés en l'état de nudité complète - sous l'œil vigilant du professeur, ou de camarades, voire en situation d'autocorrection face à un miroir ou, mieux encore, entre deux miroirs. L'idée en était, à la fois, l'étude et le contrôle du jeu des muscles et des articulations (cf. n. 6).

6. - Thieß - qui, d'ailleurs, se rattache aussi à l'esprit de la " Nouvelle Objectivité» par l'écriture factuelle d'un roman comme Tsushima (1936) - était un inconditionnel du naturisme. Seule réserve : cette pratique ne suffirait pas à fonder une culture... [Cf. Thieß, Die Befreiung des Körpers, in: Idem, Erziehung zur Freiheit, Stuttgart, 1929, p. 215]. Giese est plus nuancé. Partisan de la gymnastique nue, car seule la nudité permettrait de contrôler la justesse des mouvements (cf. $\mathrm{n}$. 5), il n'admet de loisirs naturistes que pour les ouvriers affamés de plein air [cf. Giese, Girlkultur, Munich, 1925, p.116, et Geist im Sport, Munich, 1925, p.113]. Par ailleurs, il récuse la nudité sculpturale - «culturiste ", dirait-on aujourd'hui - à travers une analyse - très sartrienne, avant la lettre - de la dépossession de soi sous le regard d'autrui ; mais, paradoxalement, il ne trouve rien à redire contre la danse nue "de haut niveau artistique", sous prétexte qu'alors ses spectateurs n'« objectiveraient " pas les choreutes de leurs regards, mais communieraient autour d'un spectacle, accédant au «mystère » [cf. Idem., Körperseele, Munich, 1927, p.123, et ibid., p. 182].

7. - Cf. Graaz, Freikörperkultur [FKK] u. Lebensreform, in: Körperbildung / Nacktkultur [K/N], Sonderheft 15 [Nackt - aber warum?], 1932.

8. - Cf. Koch, Körperkultur als Mittel zur Persönlichkeitsbildung, in : K/N, erste Folge [Schrei des Volkes nach Licht], juillet 1925.

9. - Cf. Hermann Haß, Sitte u. Kultur im Nachkriegsdeutschland, Hambourg, 1932, p. 100.

10. - Cf. Thieß, Auseinandersetzungen mit einem Geistigen über «Die Geistigen u. der Sport» [1925], in : Idem, Erziehung..., p. 371, et Giese, Geist..., p. 39.

11. - Cf. Spitzer, op. cit., p. 106.

12. - Cf. Surén, Der Mensch u. die Sonne, Stuttgart, 1924, p. 107, et Idem, Gymnastik f Heim, Beruf u. Sport, Stuttgart, 1929, p. 213, n. 1. 
13. - Cf. Mülhause-Vogeler, Dürfen Nationalsozialisten FKK treiben?, in : LichtLand [LL], 1930, $\mathrm{n}^{\circ} 21$. Eadem, FKK u. Rasse, in : LL, 1931, $\mathrm{n}^{\circ}$ 6. Eadem, Weltfriedenidee u. FKK, in : LL, 1931, $\mathrm{n}^{\circ} 17$.

14. - Cf., entre autres, ${ }^{* * *}$, Chez les camarades en France méridionale, in : LL, 1931, $\mathrm{n}^{\circ}$ 8. - L'article (bilingue) relate le voyage qu'une délégation de la «Ligue » effectua, en février 1931, auprès des «naturistes de Provence». De tels contact devaient aboutir rapidement à la création de structures internationales.

15. - Cf. ***, Auch eine Einheitsfront, in : LL, 1929, $\mathrm{n}^{\circ} 19$.

16. - La "Nouvelle Objectivité », qui répondait assurément à une authentique aspi-ration à la "désidéologisation», par refus, à la fois, de l'impérialisme wilhelminien et des troubles révolutionnaires «à la soviétique » de 1918-1919, n'en devint pas moins une mode intellectuelle, voire un «label de marque ", contribuant au succès de toutes sortes de "produits ", du service à thé en pyrex de Wilhelm Wagenfeld au célèbre foxtrott de Marcellus Schiffer : «Es liegt in der Luft eine Sachlichkeit, eine Stachlichkeit, was Idiotisches, was Erotisches... » - On a même pu prétendre, à ce titre, que la «Nouvelle Objectivité » avait été happée par le "rush sur les nouveautés » [cf. Jost Hermand et Frank Trommler, Die Kultur der Weimarer Republik, Munich, 1978, p. 92].

17. - Cf. Surén, Der Mensch..., p. 32.

18. - Il ne faudrait cependant pas croire que c'est là le principal argument, à l'époque, en faveur du nu intégral. Nos auteurs et rédacteurs se prévalent surtout d'arguments psychologiques, moraux et sociaux pour exiger jusqu'à la suppression du moindre morceau de tissu. Cf. infra.

19. - Mülhause-Vogeler, Freie Lebensgestaltung, Egestorf, 1926, p. 17.

20. - Cf. Graaz, opusc. cit., loc. cit.

21. - Cf. extraits d'un développement de Graaz, reproduits dans : Koch, Nacktheit, Körperkultur u. Erziehung (1929), Berlin-Ouest, 1983, p. 110.

22. - Cf. Teßmann, Körperkultur u. ihre volkswirtschaftliche Bedeutung, in : Sorna, vol. 2, $\mathrm{n}^{\circ} 7$.

23. - Cf. Surén, Der Mensch..., p. 33.

24. - Cf. Graaz, opusc. cit., loc. cit.

25. - Cf. Surén, Der Mensch..., p. 10, et Mülhause-Vogeler, Freie Lebensgestaltung, p. 15.

26. - Cf. Ludwig Hoffmann, Es geht ein Raunen durch die Welt, in : LL, 1930, $\mathrm{n}^{\circ} 2$.

27. - Cf. Graaz, opusc. cit., loc. cit., et Giese, Geist..., p. 113.

28. - Cf. Figaro, 1929, nº 19.

29. - Cf. $L L, 1930, \mathrm{n}^{\circ} 17$.

30. - Cf. Teßmann, opusc. cit., loc. cit.

31. - Cf. Hermand et Trommler, op. cit., p.77, et, pour une critique contemporaine de cette «stratégie capitaliste », Siegfried Kracauer, Die Angestellten [1930], Francfort, 1980, p. 74 sqq. et 99 sqq.

32. - Cf. Graaz, opusc. cit., loc. cit.

33. - Cf. ibid. - Cette perméabilité de la "gauche" politique, représentée ici par Graaz et la Kochschule, aux doctrines eugéniques n'est pas une spécialité allemande à l'époque. On constate le même phénomène en France. [Cf. Lion Murard et Patrick Zylbermann, L'Ordre et la règle L'hygiénisme en France dans l'entre-deux-guerres, in : Institut Français d'Architecture (édit.), Textes préparatoires (au colloque international des 3, 4, 5/12/1981, sur: Architecture et politiques sociales), p. 124 sq.].

34. - Cf. Surén, Der Mensch..., p. 117 sq.

35. - Cf. extraits d'un développement de Graaz, reproduits dans : Koch, Nacktheit..., p. 110.

36. - Cf. Graaz, opusc. cit., loc. cit.

37. - Cf. ibid., et Mülhause-Vogeler, Freie Lebensgestaltung, p. 45.

38. - Cf. Koch, Nacktheit..., p. 106.

39. - Cf. Mülhause-Vogeler, Freie Lebensgestaltung, p. 31.

40. - Cf. Eadem, Frauen heraus!, in : LL, 1929, $\mathrm{n}^{\circ} 1$. 
41. - Cf. Hermand et Trommler, op. cit., p. 87.

42. - Cf. Mülhause-Vogeler, Freie Lebensgestaltung, p. 33.

43. - Cf. Thieß, Über die Jugend [1927], in : Idem, Erziehung..., p. 162.

44. - Cf. Thieß, Vom sexuellen Schatten der Nacktheit, in : Idem, Erziehung..., p. 295.

45. - Cf. Surén, Der Mensch..., p. 120.

46. - Cf. Thieß, Vom sexuellen Schatten der Nacktheit, loc. cit., p. 297.

47. - Cf. Hoffmann, opusc. cit., loc. cit.

48. - Cf. Surén, Gymnastik..., p. 214.

49. - Cf., respectivement, Mülhause-Vogeler, FKKu. Rasse, loc. cit. ; Thieß, Vom sexuellen Schatten der Nacktheit, loc. cit., p. 296 ; Haß, op. cit., p. 98 [citation extraite de la revue Lachendes Leben, du même groupe de presse que LL, vol. 3, $\mathrm{n}^{\circ} 7$ ] ; Rudolf Karl, Die Gemeinschaft, in : Sorna, vol. 2, $\mathrm{n}^{\circ} 7$.

50. - Cf. citation mentionnée à la n. 49.

51. - Cf. Hoffmann, opusc. cit. loc. cit.

52. - Cf. Kracauer, Kult der Zerstreuung, in : Idem, Das Ornament der Masse, Francfort, 1977, p. 313.

53. - Cf. Karl, opusc. cit., loc. cit.

54. - Cf. Koch, Nacktheit..., p. 9.

55. - Cf. Graaz, opusc. cit., loc. cit.

56. - Cf. Karl, opusc. cit., loc. cit.

57. - Cf. Graaz, Die Badehose, ein moderner Aberglaube, in : $K / N$, Sonderheft 7 [Nackt, ein Bilderbuch $f$. groß u. klein], 1929.

58. - Cf. Karl, opusc. cit., loc. cit.

59. - Cf. Koch, Nacktheit..., p. 113.

60. - Cf. Otto Weber, Sozialistische Körperkultur?, in : K/N, Sonderheft 1 [Schrei nach Licht], 1931, et Koch, Nacktheit..., p. 113.

61. - Cf. Koch, Unser Weg zur freien Liebe, in : K/N, Dritte Folge [Freie Liebe oder Zwangsehe], 1926.

62. - Cf. Idem, Nacktheit..., p. 54, et surtout Surén, Gymnastik..., p. 135 sqq.

63. - Cf. ibid., p. 141. Egalement Hans H. Reinsch, Schafft Ordnung in das Geschlechtsleben der reifen Ehelosen!, in : Figaro, $1929, \mathrm{n}^{\circ} 7$.

64. - Ibid.

65. - Teßmann, opusc. cit., loc. cit.

66. - Mülhause-Vogeler, Freie Lebensgestaltung, p. 12.

67. - Burghardt, dito, in : K/N, Sonderheft 14 [Körper u. Kunst], 1932.

68. - Cf., par exemple, extrait de : Hannes Küpper et Maxim Valentin, Die Sache ist die [1924], reproduit dans : Helmut Lethen, Neue Sachlichkeit 1924-1932, Stuttgart, 1975, p. 65.

69. - Cf. Marcuse, Vers la libération [Towards Liberation, 1969], Paris, 1970, p. 63 sqq.

70. - Cf. Lethen, op. cit., plus particulièrement p. 10 et p. 61.

71. - Cf. ibid., p. 184, n. 15.

72. - Cf. Walther Müller-Wulckow, Bauten der Gemeinschaft, Königstein im Taunus, 1929, passim.

73. - Cf.***, Wider die sündhaften fleischfarbenen Strümpfe et***, Von der Modekommission des Gesamtbundes, in : Figaro, 1929, nº 12.

74. - Cf. Ferd. Timpe, Deutscherldealismus oder Neue Sachlichkeit ?, in : Figaro, 1929, nº 14.

75. - Une collection de photos d'amateur récemment publiée tend à en confirmer l'existence [cf. Ulf Erdmann Ziegler, Die nackte Utopie, in : Zeitmagazin, 1989, $\left.\mathrm{n}^{\circ} 34\right]$.

76. - Cité dans : Luise Vierke, Wie wandern!, in : Sorna, vol. $2, \mathrm{n}^{\circ} 7$.

77. - Cf. Schacht, Vom Ende der Reparationen, Oldenburg, 1931, p. 38.

78. - Cf. Klaus Theweleit, Männerphantasien, 1.1, Francfort, 1977, p. 548 sq. Egalement Marc Cluet, L'Architecture du III'Reich, Berne, 1987, p. 215 sqq. 


\section{RÉSUMÉS}

Dans les années vingt, le naturisme, élitiste et confidentiel, d'avant-guerre sort au grand jour et se popularise. Pas un récit de voyage en Allemagne, français, anglais ou américain, qui ne signale ce phénomène de masse. Pareille transformation quantitative et qualitative s'accompagne d'un renouvellement de l'argumentaire pro-naturiste: il est de moins en moins question de virilité conquérante ou de charmes féminins qu'il faudrait arracher au corset, et toujours davantage, d'hygiène individuelle et sociale. Surtout, l'argumentation s'enrichit de considérations économiques des plus variées dans le détail : depuis l'épargne réalisée sur la garde-robe jusqu'au rendement accru du naturiste à son poste de travail. Partant de ces observations, l'article pose le problème de la perméabilité du mouvement naturiste de l'entre-deux-guerres aux théories de la " Nouvelle Objectivité », et essaie d'en décrire les modalités. Outre les valeurs caractéristiques de la santé, de l'efficacité et du bien-être, on découvre les grandes lignes d'un véritable projet de « démocratie naturiste».

In den zwanziger Jahren tritt die elitäre und öffentlichkeitsscheue Freikörperkultur der Vorkriegszeit ins gesellschaftliche Licht und erweitert ihre soziologische Basis. Kaum ein Reisebericht aus Deutschland, ob nun von französischer, englischer oder amerikanischer Hand, der nicht auf das Massenphänomen einginge. Diese quantitative und qualitative Entwicklung hat in der gewandelten Argumentationsstrategie der FKK-Befürworter ihre Entsprechung: Es ist immer weniger von männlichem Heldentum die Rede, oder von weiblichen Reizen, die dem Korsettzwang entrissen werden müßten, aber immer mehr von Individual- und Sozialhygiene. Vor allem bereichert sich die Argumentation um wirtschaftliche Gesichtspunkte, die in der Detaildurchführung größte Vielfalt aufweisen: Von der Verminderung der Kleidungskosten bis zur verstärkten Leistung des FKK-Anhängers an seinem Arbeitsposten. Von diesen Beobachtungen ausgehend stellt der Artikel das Problem der Empfänglichkeit der FKK-Reihen gegenüber den Theorien der «Neuen Sachlichkeit» in der Zeit zwischen den Weltkriegen und versucht, deren Einwirkung daselbst genau zu beschreiben. Über die charakteristischen Werte der Gesundheit, der Leistungsfähigkeit und des Wohlbefindens hinaus läßt sich in der Tat das Gesellschaftsideal einer «nudistischen Demokratie» in groben Umrissen erkennen.

\section{AUTEUR}

\section{MARC CLUET}

Université de Paris-Sorbonne 